\title{
Clinicopathological Analysis of Non-B Non-C Hepatocellular Carcinoma Focusing on Cellular Proliferation
}

\author{
SHINJI MIZUOCHI ${ }^{1}$, JUN AKIBA ${ }^{2}$, REIICHIRO KONDO ${ }^{1}$, HIRONORI KUSANO $^{1,3}$, \\ TARO SHIOGA $^{1}$, KEIICHI KONDO ${ }^{1}$, KANA TSUTSUI $^{1}$, MASAMICHI NAKAYAMA ${ }^{1}$, \\ SACHIKO OGASAWARA ${ }^{1}$, YOSHIKI NAITO ${ }^{2}$, OSAMU NAKASHIMA ${ }^{4}$ and HIROHISA YANO ${ }^{1}$ \\ ${ }^{1}$ Department of Pathology, Kurume University School of Medicine, Kurume, Japan; \\ ${ }^{2}$ Department of Diagnostic Pathology, Kurume University Hospital, Kurume, Japan; \\ ${ }^{3}$ Department of Clinical Laboratory, National Hospital Organization Kokura Medical Center, Kitakyushu, Japan; \\ ${ }^{4}$ Department of Clinical Laboratory Medicine, Kurume University Hospital, Kurume, Japan
}

\begin{abstract}
Background/Aim: Non-B non-C hepatocellular carcinomas (NBNC-HCCs) are larger than hepatitis virusrelated HCCs. We conducted a clinicopathological study of patients who underwent curative NBNC-HCC resection, including proliferative activity assessments, such as nuclear grade and Ki-67 labelling index (LI). Materials and Methods: Histopathological findings of 197 patients were examined, including 56 NBNC-HCCs, 45 hepatitis B virus (HBV)-related HCCs (HBV-HCC), and 96 hepatitis C virus (HCV)-related HCCs (HCV-HCC). Results: NBNC-HCCs were significantly larger than HCV-HCCs, but not significantly different from HBV-HCCs. Mitotic counts, nuclear grade, and Ki-67 LI of NBNC-HCCs were not significantly different from those of $H C V$-HCCs, but were significantly lower than those of $\mathrm{HBV}$ $H C C s$. Recurrence-free survival was significantly better in the NBNC-HCC group than in the HBV-HCC group in cases with mild liver fibrosis. Conclusion: NBNC-HCCs were significantly larger in diameter, but their nuclear grade or Ki-67 LI were not significantly different from those of other HCCs, suggesting that they do not have a higher proliferative activity.
\end{abstract}

The most important aetiological factor associated with hepatocellular carcinoma (HCC) worldwide is persistent infection with either hepatitis C virus (HCV) or hepatitis B

This article is freely accessible online.

Correspondence to: Shinji Mizuochi, Department of Pathology, Kurume University School of Medicine, Asahi-machi 67, Kurume, 830-0011, Japan. Tel: +81 942317546, Fax: +81 942320905, e-mail: mizuochi_shinji@kurume-u.ac.jp

Key Words: Non-B non-C hepatocellular carcinoma, cellular proliferation, tumor size, ki-67. virus (HBV). Among patients in Japan, persistent $\mathrm{HCV}$ infection is the leading cause of $\operatorname{HCC}(1,2)$. The development of antiviral therapies has led to a relative decline in $\mathrm{HCC}$ caused by $\mathrm{HCV}$ and $\mathrm{HBV}$ infections. However, an increase in non-B non-C hepatocellular carcinoma (NBNC-HCC), which is negative for both $\mathrm{HBV}$ surface antigen ( $\mathrm{HBs} \mathrm{Ag}$ ) and $\mathrm{HCV}$ antibodies (HCV Ab), is becoming a problem $(3,4)$.

Basic and clinical research regarding $\mathrm{HCC}$ has mostly focused on patients with a background of HBV- or HCVrelated chronic hepatitis (5), with treatment and screening algorithms being based on these patient types. The exact background and molecular mechanisms underlying the rapid increase in the incidence of NBNC-HCC remain unclear; however, non-alcoholic steatohepatitis (NASH) and metabolic syndrome have been reported to be important risk factors (3).

Several recent studies have examined the prognosis of surgical cases of NBNC-HCC and hepatitis virus-related HCC, but the findings have been inconsistent with some showing no significant difference in prognosis, whereas others indicating that patients with NBNC-HCC have a longer disease-free survival period (6-9). A study of tumour size showed that NBNC-HCCs are larger than hepatitis virusrelated HCCs (9). One possible reason for this finding is the lack of proper screening, often resulting in the diagnosis of the disease in an advanced state; moreover, research regarding the proliferative activity of HCCs is limited (10).

In the present study, we investigated why NBNC-HCCs are larger than hepatitis virus-related HCCs using patients with HCC who underwent curative resection as the initial therapy.

\section{Materials and Methods}

Patients. The study involved 197 patients with HCC who underwent hepatectomy at Kurume University Hospital, Kurume, Japan, between January 2008 and December 2013. The exclusion criteria 
were as follows: 1) previous treatment for HCC, 2) presence of multiple nodules before surgery, and 3) having autoimmune hepatitis, primary biliary cholangitis, or Japanese schistosomiasis. The clinicopathological characteristics of the 197 patients are shown in Table I. The 197 patients comprised 56 patients with NBNC-HCC (28.4\%), 45 with HBV-HCC (22.8\%), and 96 with HCV-HCC $(48.7 \%)$. The study was approved by the Kurume University Institutional Review Board (approval No. 20116) and complied with the guidelines of the Declaration of Helsinki.

Histopathological examination. Resection specimens consisting of HCC and non-neoplastic tissue were collected from patients who underwent hepatectomy, fixed with $10 \%$ buffered formalin, and embedded in paraffin to obtain tissue blocks. The blocks were cut to sections of $4 \mu \mathrm{m}$ thickness; then, the sections were stained with haematoxylin and eosin, and evaluated by two pathologists (SM and JA). The specimens were assessed for diameter, histological differentiation, histological subtype, portal vein invasion, intrahepatic metastatic nodules, nuclear grade, and mitotic count of the tumour as well as fibrosis, inflammation, and fatty changes in non-neoplastic tissues. Tumour subtypes were evaluated according to the World Health Organization (WHO) classification 2019 (11). Histological atypia and mitotic count were evaluated according to nuclear grade (NG), which is widely used in the pathological diagnosis of breast cancer (Figure 1, Table II) (12). NG was determined by adding nuclear atypia and mitotic count scores (12). Fibrosis and inflammation in non-neoplastic tissues were assessed according to Batts-Ludwig staging and grading (13). Fibrosis based on stage was classified as none (stage 0), mild (portal fibrosis, stage 1), moderate (periportal fibrosis, stage 2), severe (bridging fibrosis with lobar distortion, stage 3), and cirrhosis (stage 4). The patients were classified as those with mild fibrosis $(\mathrm{F}=0, \mathrm{~F}=1$, or $\mathrm{F}=2)$ or those with severe fibrosis $(\mathrm{F}=3$, or $\mathrm{F}=4)$. Inflammation grade according to the degree and location of the inflammation was classified as none (grade 0), minimal (grade 1), mild (grade 2), moderate (grade 3 ), or severe (grade 4) (13). The background liver tissue in the NBNC group was evaluated for ballooned hepatocytes and large liver cell changes (LCCs). Steatosis was defined as having $\geq 5 \%$ large and small lipid droplets, and steatohepatitis was defined as steatosis plus ballooned hepatocytes and lobular inflammation. Finally, NASH was defined as the absence of a history of alcohol consumption.

Tissue microarray (TMA) preparation. TMA was used to perform multiplex histological analysis of the tissue specimens. The TMA blocks were prepared using an arraying instrument (Azumaya, Tokyo, Japan). Two 4-mm diameter cores were punched from the tumour portion of the "donor" paraffin-embedded tissue block for each patient included in the study and placed onto "recipient" TMA blocks. Each TMA block contained 22 cores.

Immunohistochemical analysis. Slides were created using 4- $\mu \mathrm{m}$ thick sections cut from the TMA paraffin blocks and labelled with anti-Ki-67 antibody (NCL-Ki67-MM1, dilution 1:200; Leica Biosystems, Nussloch, Germany) using a BenchMark ULTRA slide staining system (Ventana Automated Systems Inc., Tucson, AZ, USA). The Ki-67 labelling index (LI) was calculated as the percentage of tumour cells with positively stained nuclei in the region of tissue with the highest expression of Ki-67 (hotspot).

Statistical analysis. All statistical analyses were performed using JMP Pro 13.21 software (SAS Institute Inc., Cary, NC, USA).
Table I. Clinicopathological characteristics of 197 patients who underwent hepatectomy for $\mathrm{HCC}$.

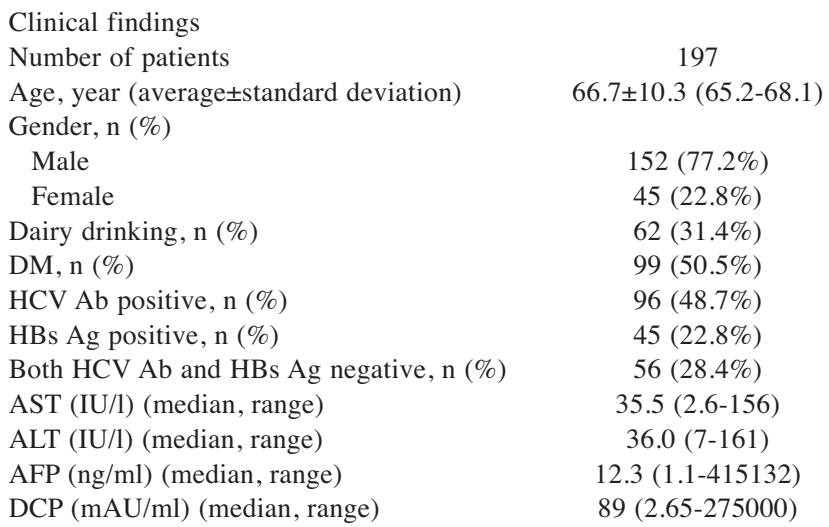

Number of patients

Age, year (average \pm standard deviation)

Gender, n (\%)

Male

Female

Dairy drinking, $\mathrm{n}(\%)$

$\mathrm{DM}, \mathrm{n}(\%)$

$\mathrm{HCV}$ Ab positive, $\mathrm{n}(\%)$

HBs Ag positive, n (\%)

Both $\mathrm{HCV} \mathrm{Ab}$ and HBs Ag negative, $\mathrm{n}(\%)$

AST (IU/l) (median, range)

ALT (IU/l) (median, range)

AFP (ng/ml) (median, range)

$\mathrm{DCP}(\mathrm{mAU} / \mathrm{ml})$ (median, range)

$66.7 \pm 10.3(65.2-68.1)$

$152(77.2 \%)$

$45(22.8 \%)$

$62(31.4 \%)$

$99(50.5 \%)$

$96(48.7 \%)$

$45(22.8 \%)$

$56(28.4 \%)$

$35.5(2.6-156)$

$36.0(7-161)$

$12.3(1.1-415132)$

$89(2.65-275000)$

Pathological findings

Tumour size, $\mathrm{mm}$ (average \pm standard deviation)

$35.6 \pm 26.7$

Tumour differentiation

Well

Moderately

$15(7.61 \%)$

Poorly

Portal vein invasion, $\mathrm{n}(\%)$

Intrahepatic metastasis, $\mathrm{n}(\%)$

Fibrosis, n (stage: 0/1/2/3/4)

Inflammation, $\mathrm{n}$ (activity: 0/1/2/3)

$144(73.1 \%)$

$38(19.3 \%)$

$119(60.4 \%)$

$24(12.7 \%)$

$3 / 48 / 49 / 31 / 66$

$3 / 87 / 105 / 2$

HCC: Hepatocellular carcinoma; DM: diabetes mellitus; HCV Ab: hepatitis C virus antibody; HBs Ag: hepatitis B surface antigen; AST: aspartate transaminase; ALT: alanine aminotransferase; AFP: $\alpha$ fetoprotein; DCP: des- $\gamma$-carboxy prothrombin.

Continuous variables are presented as mean \pm standard deviation. A chi-squared test or Dunnett's test was used to evaluate the discrete data. Overall survival (OS) curves and recurrence-free survival (RFS) curves were created using the Kaplan-Meier method and compared using a log-rank test. The $p$-values were corrected for multiple comparisons in all cases using Bonferroni correction following stepwise criteria. The significance level was set at $p=0.05$, and bilateral tests were performed.

\section{Results}

Comparison of clinicopathological characteristics between the $N B N C$ group and $H B V$ and HCV groups of patients. The clinicopathological features of the patients with NBNC-HCC, HBV-HCC, and HCV-HCC are shown in Table III. As this study focused on NBNC-HCC, we compared the NBNC-HCC and HBV-HCC groups and the NBNC-HCC and HCV-HCC groups, but not the HBV-HCC and HCV-HCC groups. Patients in the NBNC group on an average were significantly older than those in the HBV group $(p<0.001)$, but comparable to those in the HCV group ( $p=0.3395)$. The proportion of men in the NBNC group was higher than that in the HCV group ( $p=0.0104)$, but similar to that in the HBV group $(p=0.5234)$. 

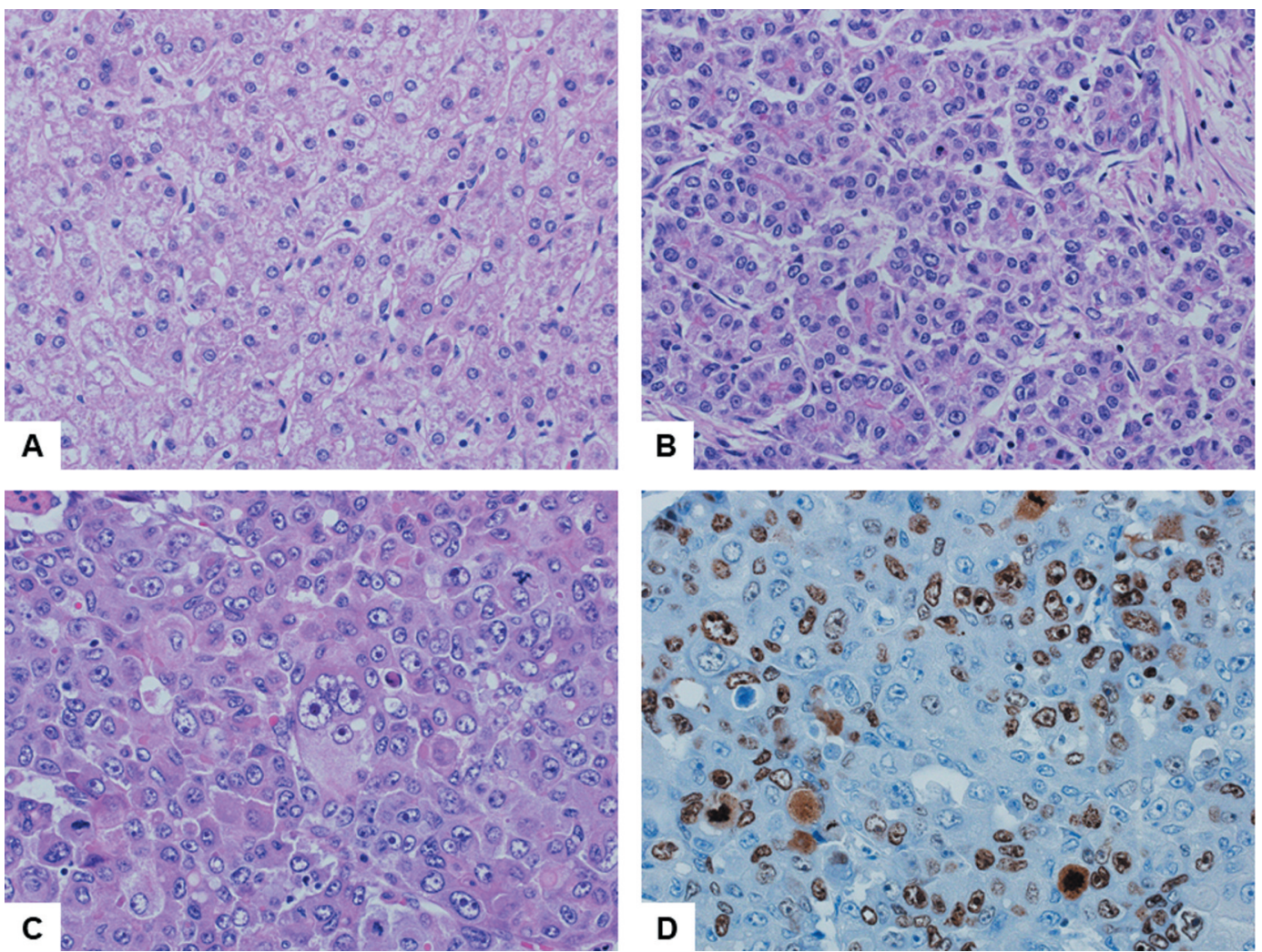

Figure 1. Representative histologic features of nuclear atypia and immunohistochemical staining of Ki-67. (A) Nuclear atypia score 1. The tumour cells have a subtle degree of nuclear pleomorphism. (B) Nuclear atypia score 2. The tumour cells exhibit a moderate degree of nuclear pleomorphism. (C) Nuclear atypia score 3. The tumour shows a marked degree of cellular pleomorphism. Multinucleated cells are observed. (D) Immunohistochemical staining of Ki-67 revealed positive nuclear staining in approximately 50\% of the tumour cells.

The aspartate transaminase (AST) level was significantly lower in the NBNC group than in the HCV group $(p=0.006)$. Meanwhile, the $\alpha$-fetoprotein (AFP) level was significantly lower in the NBNC group than in the HBV group $(p=0.0222)$. Portal vein invasion was significantly more common in the NBNC group than in the HCV group $(p=0.0348)$. Finally, tumour diameter was the largest in the NBNC group at $45.2 \pm 32.7 \mathrm{~mm}$, which was significantly larger than that in the $\mathrm{HCV}$ group $(p=0.0022)$, but not significantly different from that in the HBV group $(p=0.0575)$.

Among the HCC subtypes, steatohepatitis ( $\mathrm{SH}$ )-HCC was more common in the NBNC-HCC group than in the HBV group $(p=0.2552)$ or the HCV group $(p=0.1038)$. Lymphocyte-rich HCC tended to be less common in the NBNC-HCC group than in the HBV group, but the difference was not significant $(p=0.1720)$. Mitotic count scores, nuclear grade, and Ki-67 LI were similar in the NBNC and HCV groups ( $p=0.0888, p=1.0$, and $p=0.8624$, respectively), but were significantly lower than those in the HBV group ( $p=0.0056, p=0.0334$, and $p=0.0031$, respectively).
With respect to the background liver tissue, the NBNC group had fewer patients with severe fibrosis $(\geq F 3)$ than the HBV group ( $p=0.0006)$ and milder inflammation than the HCV group $(p=0.0002)$. The NBNC-HCC group tended to have more patients with $\geq 5 \%$ fatty changes than the $\mathrm{HBV}$ and $\mathrm{HCV}$ groups, but the differences were not significant ( $p=0.2164$ and $p=0.0772$, respectively). The LCC was significantly less in the HBV group than in the NBNC group $(p=0.0012)$ but significantly more in the $\mathrm{HCV}$ group $(p=0.0014)$.

Characteristics of patients in the NBNC-HCC group. Of the 56 patients in the NBNC group, 26 had a history of daily alcohol consumption and 12 were positive for hepatitis-B-core-antigen antibody ( $\mathrm{HBc} \mathrm{Ab})$. Steatohepatitis was diagnosed in 21 patients. Among the 21 patients, 11 patients had no history of alcohol consumption, finally defined as NASH. Eleven patients in this group had NASH with no history of alcohol consumption.

Prognosis of patients with HCC after hepatic resection. The prognosis of patients with HCC after hepatectomy is shown 
A

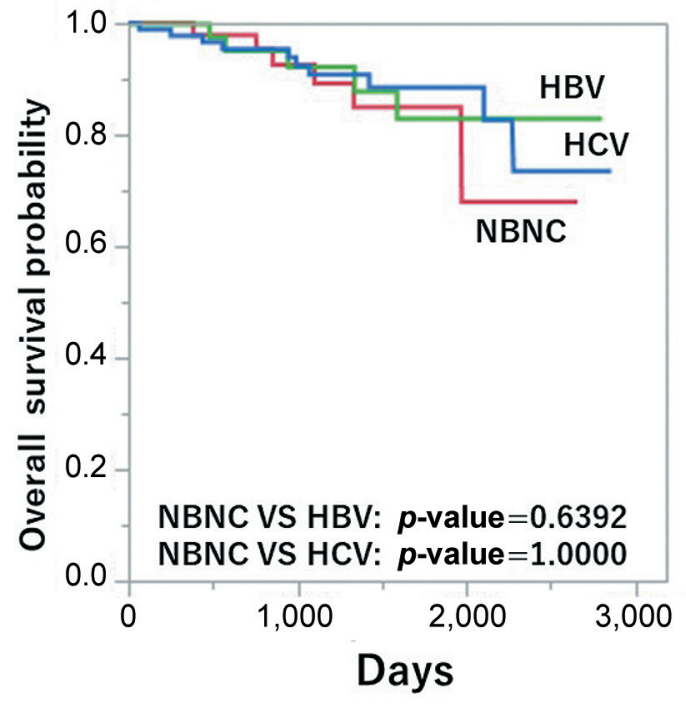

B

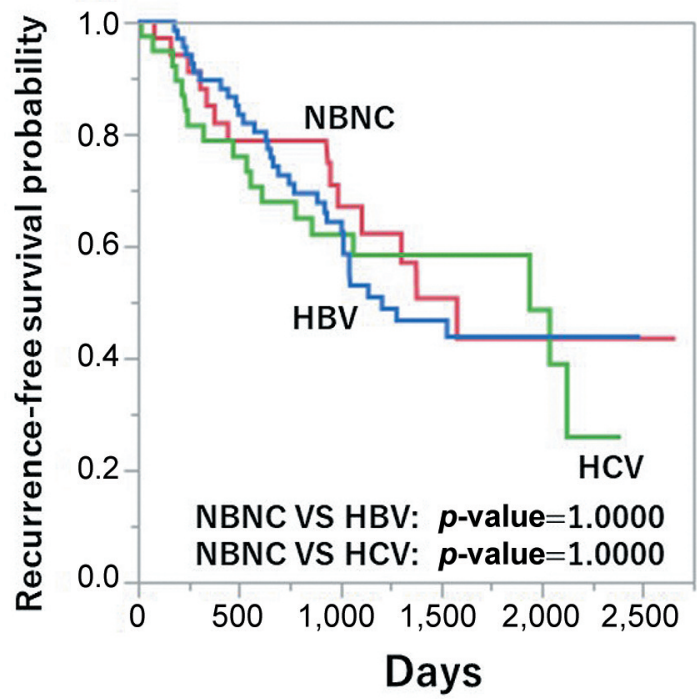

Figure 2. Comparisons of overall survival and recurrence-free survival rates after hepatectomy among patients in the $H B V-H C C, H C V-H C C$, and NBNC-HCC groups. (A) No significant difference in OS was detected between the NBNC-HCC group and HBV-HCC group (p=0.6392) or the NBNC-HCC group and HCV-HCC group $(p=1.0000)$. (B) No significant difference in recurrence-free survival was detected between the NBNCHCC group and HBV-HCC group ( $p=1.0000)$ or the NBNC-HCC group and HCV-HCC group (p=1.0000). All comparisons were made using a log-rank test. The p-values were adjusted using the Bonferroni method (2 tests). Significance level was set at p $\leq 0.05$. HBV: Hepatitis B virus; HCV: Hepatitis $C$ virus; NBNC: non-B non-C; HCC: hepatocellular carcinoma.

in Figure 2 and Figure 3. No significant differences were observed between the NBNC-HCC and HBV-HCC or HCVHCC groups regarding OS $(p=0.6392$ and $p=1.000$, respectively) or RFS ( $p=1.0000$ and $p=1.000$, respectively).

Fibrosis severity in the NBNC group compared with that in the $H B V$ and HCV groups. The severity of fibrosis in the NBNC group differed from that in the HBV and HCV groups. The RFS of patients with mild fibrosis in the NBNC-HCC group was significantly better than that in the HBV-HCC group ( $p=0.0368)$, but not significantly different from that in the HCV-HCC group $(p=0.7378)$. No significant differences according to the degree of fibrosis were observed in OS between the NBNC-HCC group and the HBV-HCC or HCV-HCC groups.

\section{Discussion}

To investigate why patients with NBNC-HCC have larger tumours than those with HBV-HCC and HBC-HCC, we analysed the clinicopathological findings for patients with NBNC-HCC and compared them with the findings for patients with HBV-HCC and patients with HCV-HCC. The analysis included assessment of tumour proliferative activity, such as nuclear grade and Ki-67 LI.

Sex and age distribution of patients with NBNC-HCC, HBV$\mathrm{HCC}$, and $\mathrm{HCV}-\mathrm{HCC}$ were similar to that previously reported
Table II. Assessment of tumour nuclear grade.

$\begin{array}{ll}\begin{array}{l}\text { A: Nuclear grade } \\ \text { Grade }\end{array} & \text { Result } \\ \text { Grade } 1 & \text { Nuclear atypia score }+ \text { Mitotic count score=2 or } 3 \\ \text { Grade 2 } & \text { Nuclear atypia score + Mitotic count score=4 } \\ \text { Grade 3 } & \text { Nuclear atypia score + Mitotic count score=5 or } 6 \\ & \\ \text { B: Nuclear atypia } & \\ \text { Score } & \text { Result } \\ \text { Score 1 } & \text { Homogenous small round nuclear cells } \\ \text { Score 2 } & \text { Score other than 1 or 3 } \\ \text { Score 3 } & \text { Multinucleated and pleomorphic cells } \\ & \\ \text { C: Mitotic count } & \\ \text { Score } & \text { Result } \\ \text { Score 1 } & <5 \text { mitotic cells/10 HPFs } \\ \text { Score 2 } & 5-10 \text { mitotic cells/10 HPFs } \\ \text { Score 3 } & >10 \text { mitotic cells/10 HPFs }\end{array}$

HPFs: High power fields.

for patients in Japan $(4-6,9,14,15)$. The mean HCC tumour diameter among the patients with NBNC-HCC was $45.2 \mathrm{~mm}$, which was significantly larger than that among the patients with HCV-HCC, similar to the results previously reported $(9,14)$.

In the present study, we used two methods to investigate the potential reason for patients with NBNC-HCC to have 

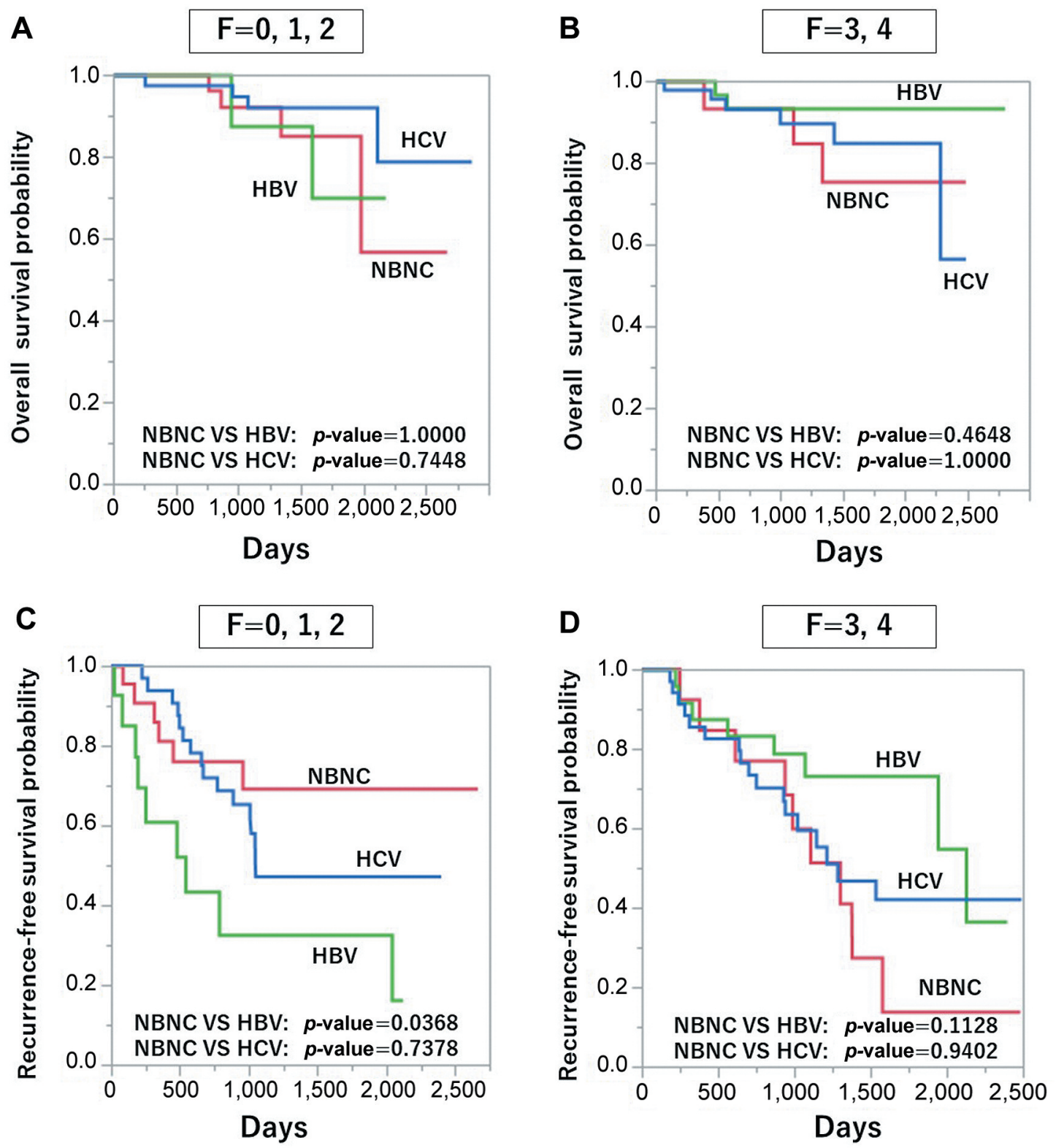

Figure 3. Comparison of overall survival (OS) and recurrence-free survival (RFS) rates after hepatectomy between patients in the NBNC-HCC group and those in the HBV-HCC and HCV-HCC groups according to fibrosis severity in the background liver tissue. The OS and RFS were stratified by fibrosis severity into stages 0,1 , and $2(A, C)$ and stages 3 and $4(B, D)$. (A) OS probability of the patients with stage 0,1 , or 2 fibrosis severity. No significant difference between the NBNC-HCC group and HBV-HCC group $(p=1.0000)$ or the NBNC-HCC group and HCV-HCC group ( $p=0.7448)$. (B) OS probability of the patients with stage 3 or 4 fibrosis severity. There was no overall significant difference between the NBNC-HCC group and $H B V-H C C$ group $(p=0.4648)$ or the NBNC-HCC group and HCV-HCC group $(p=1.0000)$. (C) RFS of the patients with stage 0,1 , or 2 fibrosis severity. RFS in the NBNC-HCC group was significantly better than that in the HBV-HCC group $(p=0.0368)$. (D) RFS with stage 3 or 4 fibrosis severity. There was no overall significant difference between the NBNC-HCC group and HBV-HCC group ( $p=0.1128)$ or the NBNC-HCC group and HCV-HCC group ( $p=0.9402)$. All comparisons were made using a log-rank test. The p-values were adjusted using the Bonferroni method (2 tests). Significance level was set as $p \leq 0.05$. HBV: Hepatitis B virus; HCV: Hepatitis C virus; NBNC: non-B non-C; HCC: hepatocellular carcinoma.

larger tumours than those with HBV-HCC or HCV-HCC. First, we assessed nuclear atypia and mitosis based on NG, which is widely used in the pathological diagnosis of breast cancer (12). The results showed similar nuclear atypia and mitotic count scores for patients with NBNC-HCC and for those with HCV-HCC. In contrast, the HBV-HCC group had a significantly higher mean mitotic count score than the NBNC-HCC group. 
Table III. Comparison of clinicopathological characteristics among patients with NBNC-HCC, HBV-HCC, and HCV-HCC.

\begin{tabular}{|c|c|c|c|c|c|}
\hline & NBNC-HCC & $\mathrm{HBV}-\mathrm{HCC}$ & $\mathrm{HCV}-\mathrm{HCC}$ & $p$-Value* & $p$-Value** \\
\hline Number of patients & 56 & 45 & 96 & & \\
\hline \multicolumn{6}{|l|}{ Clinical findings } \\
\hline Age, year (average \pm standard deviation) & $68.8 \pm 7.8$ & $55.7 \pm 10.1$ & $70.6 \pm 7.82$ & $<0.001$ & 0.3395 \\
\hline Gender, n (\%) & & & & 0.5234 & 0.0104 \\
\hline Male & $50(89.2 \%)$ & $36(80.0 \%)$ & $66(68.8 \%)$ & & \\
\hline Female & $6(10.7 \%)$ & $9(20.0 \%)$ & $30(31.3 \%)$ & & \\
\hline $\mathrm{DM}, \mathrm{n}(\%)$ & $30(53.6 \%)$ & $17(37.8 \%)$ & $52(54.2 \%)$ & 0.3198 & 1.0 \\
\hline AST (IU/L) (average \pm standard deviation) & $37.6 \pm 22.3$ & $36.9 \pm 22.3$ & $50.0 \pm 25.8$ & 0.9854 & 0.006 \\
\hline ALT (IU/L) (average \pm standard deviation) & $37.8 \pm 22.4$ & $38.8 \pm 17.9$ & $46.0 \pm 27.7$ & 0.9703 & 0.0921 \\
\hline $\mathrm{AFP}>100(\mathrm{ng} / \mathrm{ml}), \mathrm{n}(\%)$ & $13(23.2 \%)$ & $22(48.9 \%)$ & $17(17.7 \%)$ & 0.0222 & 0.8166 \\
\hline $\mathrm{DCP}>500(\mathrm{mAU} / \mathrm{ml}), \mathrm{n}(\%)$ & $22(39.3 \%)$ & $10(22.2 \%)$ & $25(26.0 \%)$ & 0.1722 & 0.2062 \\
\hline \multicolumn{6}{|l|}{ Pathological findings } \\
\hline Tumour size, $\mathrm{mm}$ (average \pm standard deviation) & $45.2 \pm 32.7$ & $33.9 \pm 30.2$ & $30.7 \pm 18.5$ & 0.0575 & 0.0022 \\
\hline Tumour differentiation & & & & 0.8234 & 0.3220 \\
\hline Well & $2(3.6 \%)$ & $1(2.2 \%)$ & $12(12.5 \%)$ & & \\
\hline Moderately & $45(80.3 \%)$ & $32(71.1 \%)$ & $67(69.8 \%)$ & & \\
\hline Poorly & $9(16.1 \%)$ & $12(26.7 \%)$ & $17(17.7 \%)$ & & \\
\hline \multicolumn{6}{|l|}{ Subtype } \\
\hline Conventional, n (\%) & $44(78.6 \%)$ & $31(71.1 \%)$ & $85(88.5 \%)$ & 0.9754 & 0.2134 \\
\hline Steatohepatic, n (\%) & $6(10.7 \%)$ & $1(2.2 \%)$ & $2(2.1 \%)$ & 0.2552 & 0.1038 \\
\hline Macrotrabecular massive, $\mathrm{n}(\%)$ & $5(8.9 \%)$ & $2(4.4 \%)$ & $4(4.2 \%)$ & 0.9136 & 0.5814 \\
\hline Lymphocyte-rich, n (\%) & $1(1.8 \%)$ & $5(11.1 \%)$ & $3(3.2 \%)$ & 0.1720 & 1.0 \\
\hline Others, n $(\%)$ & 0 & $5(11.1 \%)$ & $2(1.0 \%)$ & 0.0308 & 1.0 \\
\hline Nuclear atypia score & & & & 0.1222 & 1.0 \\
\hline 1 & $10(17.9 \%)$ & $3(6.67 \%)$ & $14(14.6 \%)$ & & \\
\hline 2 & $35(62.5 \%)$ & $25(55.6 \%)$ & $63(65.6 \%)$ & & \\
\hline 3 & $11(19.6 \%)$ & $17(37.8 \%)$ & $19(19.8 \%)$ & & \\
\hline Mitotic count score & & & & 0.0056 & 0.0888 \\
\hline 1 & $42(75.0 \%)$ & $19(42.2 \%)$ & $59(61.5 \%)$ & & \\
\hline 2 & $4(7.14 \%)$ & $10(22.2 \%)$ & $22(22.9 \%)$ & & \\
\hline 3 & $10(17.9 \%)$ & $16(35.6 \%)$ & $15(15.6 \%)$ & & \\
\hline Nuclear grade & & & & 0.0334 & 1.0 \\
\hline 1 & $37(66.1 \%)$ & $18(40.0 \%)$ & $56(58.3 \%)$ & & \\
\hline 2 & $8(14.3 \%)$ & $7(15.6 \%)$ & $18(18.8 \%)$ & & \\
\hline 3 & $11(19.6 \%)$ & $20(44.4 \%)$ & $22(22.9 \%)$ & & \\
\hline Ki-67 Labelling index, $\%$ & $8.41 \pm 15.3$ & $15.8 \pm 19.1$ & $7.25 \pm 11.7$ & 0.0031 & 0.8624 \\
\hline Portal vein invasion, $\mathrm{n}(\%)$ & $39(69.7 \%)$ & $33(73.3 \%)$ & $47(49.0 \%)$ & 1.6506 & 0.0348 \\
\hline Intrahepatic metastasis, $\mathrm{n}(\%)$ & $10(18.2 \%)$ & $5(11.3 \%)$ & $9(10.0 \%)$ & 0.8158 & 0.4102 \\
\hline Fibrosis (stage: $F \geq 3$ ) & $18(32.1 \%)$ & $31(68.9 \%)$ & $48(50.0 \%)$ & 0.0006 & 0.0834 \\
\hline Activity (activity: $A \geq 2$ ) & $17(30.4 \%)$ & $14(31.1 \%)$ & $76(79.2 \%)$ & 1.0 & 0.0002 \\
\hline Steatosis, $\mathrm{n}(\%)$ & & & & 0.2164 & 0.0772 \\
\hline$<5 \%$ & $28(50 \%)$ & $30(66.7 \%)$ & $65(67.7 \%)$ & & \\
\hline$\geq 5 \%$ & $28(50 \%)$ & $15(33.3 \%)$ & $31(33.3 \%)$ & & \\
\hline Large liver cell change, $\mathrm{n}(\%)$ & $20(35.7 \%)$ & $32(71.1 \%)$ & $11(11.5 \%)$ & 0.0012 & 0.0014 \\
\hline
\end{tabular}

*NBNC-HCC $v s$. HBV-HCC. **NBNC-HCC vs. HCV-HCC. HCC: Hepatocellular carcinoma; HBV: hepatitis B virus; HCV: hepatitis C virus; DM: diabetes mellitus; AST: aspartate aminotransferase; ALT: alanine aminotransferase; AFP: $\alpha$-fetoprotein; DCP: des- $\gamma$-carboxy prothrombin. All comparisons were made using a chi-squared test with Bonferroni correction or Dunnett's test. The $p$-values were adjusted using the Bonferroni method (two tests). Significance was set at $p \leq 0.05$.

Next, we examined the proliferative activity of tumour cells using Ki-67 immunostaining. Ki-67 is a marker expressed in all phases of the cell cycle except G0 (resting phase) and is widely used as a marker of proliferative activity in various tumours $(11,16,17)$. Although reports regarding the Ki-67 LI in HCC vary, it has been found to correlate with high histological grade, large tumour size, and the number of tumour nodes (18). In our current study, 
NBNC-HCC had a significantly larger tumour diameter than that of HCV-HCC, but its Ki-67 LI value was similar to that of HCV-HCC. This indicates that NBNC-HCC does not exhibit high proliferative activity and suggests that NBNCHCC may be less likely to be detected early, and instead may be commonly detected in more advanced states.

The mitotic count and Ki-67 LI are high in HBV-HCC because $\mathrm{HBV}$ is integrated into the host DNA during the early stages of clonal expansion, which promotes genomic instability and directs insertional mutagenesis in driver genes related to carcinogenesis (19). Furthermore, the activation of p53 and cell cycle pathways are common in HBV-HCC, which may explain why the mitotic count and Ki-67 LI were higher in this group of patients than in those in the other groups (20-22).

In the current study, we also evaluated the histological subtypes of HCC. It has been reported that SH-HCC exhibits histology similar to that of steatohepatitis, including tumourcell fat changes, ballooning, appearance of Mallory-Denk bodies, interstitial fibrosis, and infiltration of inflammatory cells (23). SH-HCC is also strongly associated with steatohepatitis and metabolic syndrome (24). Of the six patients with SH-HCC among the NBNC-HCC patients in our study, steatosis was observed in the background liver of three patients, two were with NASH. Therefore, SH-HCC was associated with steatosis/steatohepatitis, consistent with the findings of a previous study (11).

Patients with $\mathrm{HBc} \mathrm{Ab}$ positivity and $\mathrm{HBs} \mathrm{Ag}$ negativity are considered to have occult HBV infection (25). Of the 56 patients with NBNC-HCC in the present study, 35 were tested for $\mathrm{HBc} \mathrm{Ab}$ of which 12 (34.3\%) were positive. The reported frequency of $\mathrm{HBc} \mathrm{Ab}$ positivity in NBNC-HCC varies from $8 \%$ to $64 \%$ (26-29). HBV DNA integration is also detected in a significantly higher proportion in $\mathrm{HBc} \mathrm{Ab}$-positive patients with $\mathrm{HCC}$ than in $\mathrm{HBc}$ Ab-negative patients with HCC (30). Furthermore, it has been reported that the HBV genome is frequently incorporated into the tumour and background liver cells of patients with NBNC-HCC, even if serum $\mathrm{HBc} \mathrm{Ab}$ results are negative (28). Therefore, to histologically investigate the involvement of $\mathrm{HBV}$ in $\mathrm{HCC}$, we evaluated the presence and absence of LCC, which is known to be closely associated with $\mathrm{HBV}$ infection, even though it has also been observed in various other liver disorders $(28,31-33)$. We detected LCC in $35.7 \%$ of the NBNC-HCC cases. Kondo et al. reported LCC was present in $75 \%$ of NBNC-HCC, whereas other reports indicated that LCCs are dysplastic lesions closely related to hepatocarcinogenesis or are associated with aging $(34,35)$. These findings demonstrate a lack of consensus. Overall, the significance of HBV in NBNC-HCC remains controversial and more detailed studies are required, including genetic analyses.

With respect to background liver tissue, many patients in our study with NBNC-HCC exhibited milder fibrosis and less inflammation than those with other types of HCC and often had $\geq 5 \%$ fatty changes. Fibrosis in the background liver of patients with NBNC-HCC is generally considered to be mild (9), which is consistent with our results. Among the patients in our study with NBNC-HCC, 28 (50\%) exhibited $\geq 5 \%$ fatty changes and $11(19.7 \%)$ had NASH or alcoholic steatohepatitis (ASH), which is similar to previously reported results from Japan $(15,36)$.

Several studies have investigated the prognosis of patients with NBNC-HCC after hepatectomy. The long-term prognosis of patients with NBNC-HCC is similar to that for patients with hepatitis virus-related HCC (14) and the survival rate of patients with NBNC-HCC is significantly better than that of patients with $\mathrm{HCV}-\mathrm{HCC}$, but similar to that of patients with HBV-HCC (8). Utsunomiya et al. reported that the RFS of patients with NBNC-HCC is significantly better than that of patients with HBV-HCC or HCV-HCC (9). We did not observe significant differences in OS or RFS between the NBNC-HCC group and the HBV-HCC or HCV$\mathrm{HCC}$ group. In general, the prognosis of $\mathrm{HCC}$ is determined by the degree of liver preservation in the background liver tissue along with tumour-related factors $(9,37)$. Although tumours are relatively large in patients with NBNC-HCC, liver preservation is presumed to be maintained because of the occurrence of only mild fibrosis in the background liver. It is possible that the condition of the background liver may have negated the effects of the tumour-related factors, leading to a lack of difference in prognosis compared with other aetiologies. Among patients with mild fibrosis, RFS was significantly better in the NBNC-HCC group than in the HBV-HCC group, indicating that aggressive surgical intervention would be a good choice for NBNC-HCC patients with mild fibrosis. In contrast, RFS tended to be worse in patients with NBNC-HCC and advanced fibrosis. The precise cause of this phenomenon remains unknown. The sample size of the present study was limited and the evaluation of more cases will be needed to address this issue.

The current study had some limitations. First, this was a retrospective cohort study without prospective randomisation. Second, the medical records did not indicate the amount of alcohol consumed; thus, we did not have a precise understanding of this parameter. Third, as only patients for whom surgical resection was possible were considered, there may have been selection bias towards cases with relatively low stage disease and in which the level of liver preservation was better maintained. Finally, the effects of a preoperative diet may have improved fatty changes in patients who preoperatively had a severe fatty liver.

\section{Conclusion}

The group of patients with NBNC-HCC had significantly larger tumours than those with NBV-HCC and NCV-HCC, but they did not exhibit significant differences in nuclear 
grade or Ki-67 LI, indicating the proliferative capacity was not high. The results suggest that the tumours in the NBNCHCC group may have been detected at a more advanced state than those in the NBV-HCC and NCV-HCC groups.

\section{Conflicts of Interest}

The Authors declare that they have no conflicts of interest to disclose in relation to this manuscript.

\section{Authors' Contributions}

Shinji Mizuochi, Reiichiro Kondo and Jun Akiba conceived the study. Shinji Mizuochi and Jun Akiba conducted pathological assessment. Shinji Mizuochi, Reiichiro Kondo, Hironori Kusano, Taro Shioga, Keiichi Kondo, Kana Tsutsui, Masamichi Nakayama, Sachiko Ogasawara, Yoshiki Naito and Osamu Nakashima participated in pathological diagnosis. Shinji Mizuochi and Reiichiro Kondo collected and analyzed the data. Shinji Mizuochi and Jun Akiba drafted the article. All Authors have read and approved the final version of the manuscript.

\section{References}

1 Ikai I, Arii S, Okazaki M, Okita K, Omata M, Kojiro M, Takayasu K, Nakanuma Y, Makuuchi M, Matsuyama Y, Monden M and Kudo M: Report of the 17th nationwide follow-up survey of primary liver cancer in Japan. Hepatol Res 37(9): 676-691, 2007. PMID: 17617112. DOI: 10.1111/j.1872-034X.2007.00119.x

2 Namiki I, Nishiguchi S, Hino K, Suzuki F, Kumada H, Itoh Y, Asahina Y, Tamori A, Hiramatsu N, Hayashi N and Kudo M: Management of hepatitis C; Report of the consensus meeting at the 45th annual meeting of the Japan Society of Hepatology (2009). Hepatol Res 40(4): 347-368, 2010. PMID: 20394674. DOI: $10.1111 /$ j.1872-034X.2010.00642.x

3 Utsunomiya T and Shimada M: Molecular characteristics of noncancerous liver tissue in non-B non-C hepatocellular carcinoma. Hepatol Res 41(8): 711-721, 2011. PMID: 21682827. DOI: 10.1111/j.1872-034X.2011.00818.x

4 Tateishi R, Uchino K, Fujiwara N, Takehara T, Okanoue T, Seike M, Yoshiji H, Yatsuhashi H, Shimizu M, Torimura T, Moriyama M, Sakaida I, Okada H, Chiba T, Chuma M, Nakao K, Isomoto H, Sasaki Y, Kaneko S, Masaki T, Chayama K and Koike K: A nationwide survey on non-B, non-C hepatocellular carcinoma in Japan: 2011-2015 update. J Gastroenterol 54(4): 367-376, 2019. PMID: 30498904. DOI: 10.1007/s00535-018-1532-5

5 Takenaka K, Yamamoto K, Taketomi A, Itasaka H, Adachi E, Shirabe K, Nishizaki T, Yanaga K and Sugimachi K: A comparison of the surgical results in patients with hepatitis $\mathrm{B}$ versus hepatitis C-related hepatocellular carcinoma. Hepatology 22(1): 20-24, 1995. PMID: 7601413.

6 Zhou Y, Si X, Wu L, Su X, Li B and Zhang Z: Influence of viral hepatitis status on prognosis in patients undergoing hepatic resection for hepatocellular carcinoma: a meta-analysis of observational studies. World J Surg Oncol 9: 108, 2011. PMID: 21933440. DOI: 10.1186/1477-7819-9-108

7 Kaneda K, Kubo S, Tanaka H, Takemura S, Ohba K, Uenishi T, Kodai S, Shinkawa H, Urata Y, Sakae M, Yamamoto T and Suehiro $\mathrm{S}$ : Features and outcome after liver resection for non-B non-C hepatocellular carcinoma. Hepatogastroenterology 59(118): 18891892, 2012. PMID: 22819910. DOI: 10.5754/hge10778

8 Kaibori M, Ishizaki M, Matsui K and Kwon AH: Clinicopathologic characteristics of patients with non-B non-C hepatitis virus hepatocellular carcinoma after hepatectomy. Am J Surg 204(3): 300307, 2012. PMID: 22591698. DOI: 10.1016/j.amjsurg.2011.11.014

9 Utsunomiya T, Shimada M, Kudo M, Ichida T, Matsui O, Izumi N, Matsuyama Y, Sakamoto M, Nakashima O, Ku Y, Takayama T, Kokudo N and Liver Cancer Study Group of Japan: A comparison of the surgical outcomes among patients with HBV-positive, HCVpositive, and non-B non-C hepatocellular carcinoma: a nationwide study of 11,950 patients. Ann Surg 261(3): 513-520, 2015. PMID: 25072437. DOI: 10.1097/SLA.0000000000000821

10 Li Y, Chen J, Weng S, Sun H, Yan C, Xu X, Ye R and Hong J: Small hepatocellular carcinoma: using MRI to predict histological grade and Ki-67 expression. Clin Radiol 74(8): 653.e1-653.e9, 2019. PMID: 31200932. DOI: 10.1016/j.crad.2019.05.009

11 Nagtegaal ID, Odze RD, Klimstra D, Paradis V, Rugge M, Schirmacher P, Washington KM, Carneiro F, Cree IA and WHO Classification of Tumours Editorial Board: The 2019 WHO classification of tumours of the digestive system. Histopathology 76(2): 182-188, 2020. PMID: 31433515. DOI: 10.1111/his.13975

12 Tsuda H, Akiyama F, Kurosumi M, Sakamoto G and Watanabe T: Establishment of histological criteria for high-risk nodenegative breast carcinoma for a multi-institutional randomized clinical trial of adjuvant therapy. Japan National Surgical Adjuvant Study of Breast Cancer (NSAS-BC) Pathology Section. Jpn J Clin Oncol 28(8): 486-491, 1998. PMID: 9769782. DOI: $10.1093 / \mathrm{jjco} / 28.8 .486$

13 Batts KP and Ludwig J: Chronic hepatitis. An update on terminology and reporting. Am J Surg Pathol 19(12): 1409-1417, 1995. PMID: 7503362. DOI: 10.1097/00000478-199512000-00007

14 Nishikawa H, Arimoto A, Wakasa T, Kita R, Kimura T and Osaki $\mathrm{Y}$ : Comparison of clinical characteristics and survival after surgery in patients with non-B and non-C hepatocellular carcinoma and hepatitis virus-related hepatocellular carcinoma. J Cancer 4(6): 502-513, 2013. PMID: 23901350. DOI: 10.7150/jca.6503

15 Nagaoki Y, Hyogo H, Aikata H, Tanaka M, Naeshiro N, Nakahara T, Honda Y, Miyaki D, Kawaoka T, Takaki S, Hiramatsu A, Waki K, Imamura M, Kawakami Y, Takahashi S and Chayama K: Recent trend of clinical features in patients with hepatocellular carcinoma. Hepatol Res 42(4): 368-375, 2012. PMID: 22151896. DOI: 10.1111/j.1872-034X.2011.00929.x

16 Synnestvedt M, Borgen E, Russnes HG, Kumar NT, Schlichting E, Giercksky KE, Kåresen R, Nesland JM and Naume B: Combined analysis of vascular invasion, grade, HER2 and Ki67 expression identifies early breast cancer patients with questionable benefit of systemic adjuvant therapy. Acta Oncol 52(1): 91-101, 2013. PMID: 22934555. DOI: 10.3109/0284186X.2012.713508

17 Niikura N, Masuda S, Kumaki N, Xiaoyan T, Terada M, Terao M, Iwamoto T, Oshitanai R, Morioka T, Tuda B, Okamura T, Saito Y, Suzuki Y and Tokuda Y: Prognostic significance of the Ki67 scoring categories in breast cancer subgroups. Clin Breast Cancer 14(5): 323-329.e3, 2014. PMID: 24492237. DOI: 10.1016/j.clbc.2013.12.013

18 Luo Y, Ren F, Liu Y, Shi Z, Tan Z, Xiong H, Dang Y and Chen $\mathrm{G}$ : Clinicopathological and prognostic significance of high $\mathrm{Ki}$ 67 labeling index in hepatocellular carcinoma patients: a metaanalysis. Int J Clin Exp Med 8(7): 10235-10247, 2015. PMID: 26379815. 
19 Levrero M and Zucman-Rossi J: Mechanisms of HBV-induced hepatocellular carcinoma. J Hepatol 64(1 Suppl): S84-S101, 2016. PMID: 27084040. DOI: 10.1016/j.jhep.2016.02.021

20 Schulze K, Imbeaud S, Letouzé E, Alexandrov LB, Calderaro J, Rebouissou S, Couchy G, Meiller C, Shinde J, Soysouvanh F, Calatayud AL, Pinyol R, Pelletier L, Balabaud C, Laurent A, Blanc JF, Mazzaferro V, Calvo F, Villanueva A, Nault JC, BioulacSage P, Stratton MR, Llovet JM and Zucman-Rossi J: Exome sequencing of hepatocellular carcinomas identifies new mutational signatures and potential therapeutic targets. Nat Genet 47(5): 505511, 2015. PMID: 25822088. DOI: $10.1038 / n g .3252$

21 Ahn SM, Jang SJ, Shim JH, Kim D, Hong SM, Sung CO, Baek D, Haq F, Ansari AA, Lee SY, Chun SM, Choi S, Choi HJ, Kim J, Kim S, Hwang S, Lee YJ, Lee JE, Jung WR, Jang HY, Yang E, Sung WK, Lee NP, Mao M, Lee C, Zucman-Rossi J, Yu E, Lee HC and Kong G: Genomic portrait of resectable hepatocellular carcinomas: implications of RB1 and FGF19 aberrations for patient stratification. Hepatology 60(6): 19721982, 2014. PMID: 24798001. DOI: 10.1002/hep.27198

22 Amaddeo G, Cao Q, Ladeiro Y, Imbeaud S, Nault JC, Jaoui D, Gaston Mathe Y, Laurent C, Laurent A, Bioulac-Sage P, Calderaro $\mathrm{J}$ and Zucman-Rossi J: Integration of tumour and viral genomic characterizations in HBV-related hepatocellular carcinomas. Gut 64(5): 820-829, 2015. PMID: 25021421. DOI: 10.1136/gutjnl-2013-306228

23 Salomao M, Yu WM, Brown RS Jr, Emond JC and Lefkowitch JH: Steatohepatitic hepatocellular carcinoma (SH-HCC): a distinctive histological variant of $\mathrm{HCC}$ in hepatitis $\mathrm{C}$ virusrelated cirrhosis with associated NAFLD/NASH. Am J Surg Pathol 34(11): 1630-1636, 2010. PMID: 20975341. DOI: 10.1097/PAS.0b013e3181f31caa

24 Salomao M, Remotti H, Vaughan R, Siegel AB, Lefkowitch JH and Moreira RK: The steatohepatitic variant of hepatocellular carcinoma and its association with underlying steatohepatitis. Hum Pathol 43(5): 737-746, 2012. PMID: 22018903. DOI: 10.1016/j.humpath.2011.07.005

25 Urbani S, Fagnoni F, Missale G and Franchini M: The role of anti-core antibody response in the detection of occult hepatitis B virus infection. Clin Chem Lab Med 48(1): 23-29, 2010. PMID: 19919328. DOI: 10.1515/CCLM.2010.002

26 Omichi K, Shindoh J, Yamamoto S, Matsuyama Y, Akamatsu N, Arita J, Kaneko J, Sakamoto Y, Hasegawa K and Kokudo N: Postoperative outcomes for patients with non-B non-C hepatocellular carcinoma: a subgroup analysis of patients with a history of Hepatitis B infection. Ann Surg Oncol 22 Suppl 3: S1034-S1040, 2015. PMID: 26350363. DOI: 10.1245/s10434015-4845-0

27 Okamura Y, Sugiura T, Ito T, Yamamoto Y, Ashida R and Uesaka $\mathrm{K}$ : The impact of the Hepatitis $\mathrm{B}$ core antibody status on recurrence in patients with non-B non-C hepatocellular carcinoma after curative surgery. Dig Surg 35(3): 243-251, 2018. PMID: 28810252. DOI: 10.1159/000479340
28 Kondo R, Nakashima O, Sata M, Imazeki F, Yokosuka O, Tanikawa K, Kage M, Yano H and Liver Cancer Study Group of Kyushu: Pathological characteristics of patients who develop hepatocellular carcinoma with negative results of both serous hepatitis B surface antigen and hepatitis C virus antibody. Hepatol Res 44(11): 1039-1046, 2014. PMID: 23937266. DOI: 10.1111/hepr.12219

29 Koga H, Kai K, Aishima S, Kawaguchi A, Yamaji K, Ide T, Ueda $\mathrm{J}$ and Noshiro H: Occult hepatitis B virus infection and surgical outcomes in non-B, non-C patients with curative resection for hepatocellular carcinoma. World J Hepatol 9(35): 1286-1295, 2017. PMID: 29290910. DOI: 10.4254/wjh.v9.i35.1286

30 Tamori A, Nishiguchi S, Kubo S, Narimatsu T, Habu D, Takeda T, Hirohashi K and Shiomi S: HBV DNA integration and HBVtranscript expression in non-B, non-C hepatocellular carcinoma in Japan. J Med Virol 71(4): 492-498, 2003. PMID: 14556260. DOI: $10.1002 / j m v .10514$

31 Park YN and Roncalli M: Large liver cell dysplasia: a controversial entity. J Hepatol 45(5): 734-743, 2006. PMID: 16982109. DOI: $10.1016 /$ j.jhep.2006.08.002

32 Paradis V, Laurendeau I, Vidaud M and Bedossa P: Clonal analysis of macronodules in cirrhosis. Hepatology 28(4): 953958, 1998. PMID: 9755231. DOI: 10.1002/hep.510280409

33 Libbrecht L, Craninx M, Nevens F, Desmet V and Roskams T: Predictive value of liver cell dysplasia for development of hepatocellular carcinoma in patients with non-cirrhotic and cirrhotic chronic viral hepatitis. Histopathology 39(1): 66-73, 2001. PMID: 11454046. DOI: 10.1046/j.1365-2559.2001.01172.x

34 Kim H, Oh BK, Roncalli M, Park C, Yoon SM, Yoo JE and Park YN: Large liver cell change in hepatitis B virus-related liver cirrhosis. Hepatology 50(3): 752-762, 2009. PMID: 19585549. DOI: $10.1002 /$ hep. 23072

35 Ikeda H, Sasaki M, Sato Y, Harada K, Zen Y, Mitsui T and Nakanuma Y: Large cell change of hepatocytes in chronic viral hepatitis represents a senescent-related lesion. Hum Pathol 40(12): 1774-1782, 2009. PMID: 19733384. DOI: 10.1016/ j.humpath.2009.06.009

36 Hiwatashi K, Ueno S, Sakoda M, Iino S, Minami K, Yamasaki Y, Okubo K, Noda M, Kurahara H, Mataki Y, Maemura K, Shinchi $\mathrm{H}$ and Natsugoe S: Problems of Long Survival Following Surgery in Patients with NonBNonC-HCC: Comparison with HBV and HCV Related-HCC. J Cancer 6(5): 438-447, 2015. PMID: 25874007. DOI: $10.7150 /$ jca. 10398

37 Massarweh NN and El-Serag HB: Epidemiology of hepatocellular carcinoma and intrahepatic cholangiocarcinoma. Cancer Control 24(3): 1073274817729245, 2017. PMID: 28975830. DOI: $10.1177 / 1073274817729245$

Received October 31, 2021

Revised November 23, 2021

Accepted November 24, 2021 